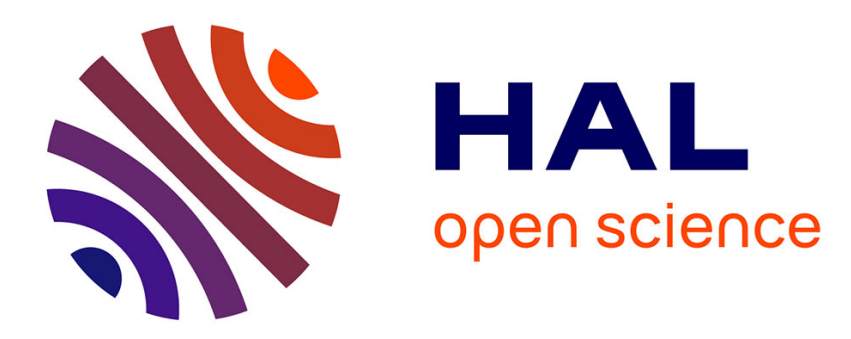

\title{
Etude génétique et amélioration des pêches plates
}

René Monet, Yves Bastard, Bertrand Gibault

\section{To cite this version:}

René Monet, Yves Bastard, Bertrand Gibault. Etude génétique et amélioration des pêches plates. Agronomie, 1985, 5 (8), pp.727-731. hal-00884805

\section{HAL Id: hal-00884805 https://hal.science/hal-00884805}

Submitted on 1 Jan 1985

HAL is a multi-disciplinary open access archive for the deposit and dissemination of scientific research documents, whether they are published or not. The documents may come from teaching and research institutions in France or abroad, or from public or private research centers.
L'archive ouverte pluridisciplinaire HAL, est destinée au dépôt et à la diffusion de documents scientifiques de niveau recherche, publiés ou non, émanant des établissements d'enseignement et de recherche français ou étrangers, des laboratoires publics ou privés. 


\title{
Etude génétique et amélioration des pêches plates
}

René MONET, Yves BASTARD \& Bertrand GIBAULT

I.N.R.A., Station d'Arboriculture fruitière, Centre de Recherches de Bordeaux, F 33140 Pont-de-la-Maye on a sélectionné deux clones pour des essais culturaux.

Mots clés additionnels : Hérédité, couleur de la chair, fruit plat, fruit doux, stérilité mâle, linkage, crossing over, sélection, nectarine plate.

\begin{abstract}
Genetic studies on the breeding of flat peaches.
In order to obtain improved « peento » varieties, the flat cultivar « Kiang $\mathrm{Si}$ » was crossed with the nectarine "Independence ». "Kiang Si » is heterozygous for the characters white flesh (Yy), saucer fruit shape (Ss) and sweet taste (Dd), while «Independence» is homozygous and recessive for these characters. The cross thus acted as a test cross. Flesh colour was found to segregate independently from fruit shape and fruit taste, but the two latter were linked ( 30 cross-over units). Both cultivars in addition being heterozygous for pollen sterility (Ps ps), this character was also found to segregate independently from the preceding ones. The $F_{2}$ gave flat nectarines. Two clones were selected among the saucer-fruit peaches for further testing.
\end{abstract}

Additional key words : Heredity, flesh colour, saucer fruit, sweet fruit, pollen sterility, linkage, crossing over, flat nectarine.

\section{INTRODUCTION}

Les pêches à forme plate ou peento sont peu connues en France. Elles font cependant l'objet d'une petite culture en Espagne et en Chine car très prisées pour leur qualité gustative.

C'est d'ailleurs de Chine qu'elles ont été introduites en Europe puis aux Etats-Unis à la fin du siècle dernier (HEDRICK, 1917). En Europe, elles n'ont fait l'objet d'aucune utilisation. Elles figurent dans les vieilles pomologies à titre de curiosité botanique (CARRIEERE, 1868). Aux Etats-Unis, au contraire, elles ont été utilisées comme géniteurs pour créer des variétés à faible repos hivernal, adaptées à des climats subtropicaux ; mais le caractère pêche plate n'a pas été retenu par les sélectionneurs. Ainsi un programme d'hybridation, commencé en 1907 en Californie, mettait en œuvre des géniteurs à long repos hivernal avec des géniteurs peento à court repos hivernal; ce programme a donné en 1933 la variété « Babcock»à fruits ronds (WELDON \& LESLEY, 1933). Cette dernière est à l'origine des variétés «Robin » et « Redwing " largement cultivées en France de nos jours.

En outre, LESLEY (1939) a observé le mode de transmission génétique du caractère "fruit plat " et montré qu'il était dominant et monogénique (symbole $\mathrm{S}$, s).

Quels sont les principaux caractères de ces pêchers ? Les arbres sont vigoureux mais souvent peu fertiles; ce manque de fertilité est probablement lié à la forme du pistil. Nous avons pu observer que les individus homozygotes dominants pour le caractère fruit plat, peu fertiles, ont un pistil très court et trapu ; les hétérozygotes qui donnent aussi des fruits plats ont un pistil de taille normale et une bonne fertilité. Les variétés qui seront sélectionnées devront donc être hétérozygotes pour ce caractère. 
Le grossissement des parois de l'ovaire après la fécondation se fait normalement sur le plan équatorial du fruit alors qu'il est réduit entre le pédoncule et le style. Le fruit mûr est ainsi relativement plat, le noyau lui-même est plat et contient une graine peu développée qui germe mal.

La zone d'attache du style à l'ovaire est fragile, elle présente souvent des craquelures. L'épiderme est peu coloré, verdâtre. Tout ceci fait que l'aspect visuel de ces fruits est médiocre.

La chair peut être ferme (caractère pavie) ou fondante, avec un noyau libre ou adhérent. La qualité de la chair est souvent excellente. Ceci tient au fait que la plupart des variétés à fruit plat présentent aussi le caractère «fruit doux ». Ce caractère à hérédité simple, dominant et monogénique (symboles $D, d$ ) se traduit par la disparition quasi totale de l'acide malique dans le jus du fruit (MONET, 1979).

L'originalité de ces pêches plates, leur qualité gustative, avaient attiré notre attention. Ne fallait-il pas tenter d'en améliorer la présentation et la fertilité pour offrir aux consommateurs un produit nouveau ? Dès 1975, nous avons entrepris l'étude génétique et l'amélioration de ce matériel ; nous nous proposons ici de présenter les premiers résultats de ce travail.

\section{LES GÉNITEURS ET LEURS DESCENDANTS PAR AUTOFÉCONDATION}

Le géniteur femelle à pêches plates que nous avons utilisé au départ est la variété «Kiang Si », probablement originaire de cette province chinoise située entre les $26^{\circ}$ et $28^{\circ}$ parallèles nord. C'est un arbre à petits fruits plats, à chair blanche, douce. Nous ne possédions en 1975 que ce géniteur ; notre base génétique était donc étroite. Pour mieux connaître cette variété, nous l'avons autofécondée et avons finalement obtenu 22 arbres dont 17 seulement ont produit des fruits. Nous avons pu nous rendre compte, grâce à cette descendance, que le géniteur " Kiang Si » était hétérozygote pour 4 caractères mendéliens connus chez le pêcher : la stérilité mâle, la couleur de la chair, le caractère " fruit plat " et le caractère " fruit doux ». En effet, l'autofécondation a fait réapparaître les formes allèles récessives de ces caractères. Le tableau 1 donne la répartition des individus pour chacun d'eux.

La disjonction fruit plat-fruit rond est biaisée (probabilité du $\chi^{2}=0,04$ ) avec un déficit d'arbres à fruits plats, sans doute à cause du peu de fertilité déjà signalé des homozygotes à fruit plat. De même, la disjonction fruit doux-fruit acide a un $\chi^{2}$ dont la probabilité est relativement faible $(0,33)$ sans qu'il soit possible de donner une explication satisfaisante à cet ajustement médiocre.

Quoi qu'il en soit, cette situation était peu favorable pour analyser les disjonctions de ces caractères associés 2 à 2 afin de détecter d'éventuelles liaisons ; nous ne ferons donc pas cette analyse. Retenons cependant la formule génotypique de la variété «Kiang $\mathrm{Si}$ » pour ces caractères mendéliens : Psps Yy Ss Dd, les symboles utilisés étant ceux donnés par le premier auteur qui a étudié le caractère, ou à défaut, ceux proposés par BAILEY \& FRENCH
TABLEAU 1

Disjonction des caractères stérilité mâle, couleur de la chair, forme du fruit, saveur du fruit, par autofécondation de la variété «Kiang Si ».

Segregation of the characters pollen sterility, flesh colour, fruit shape and fruit taste on self pollination of cultivar « Kiang Si $»$.

\begin{tabular}{|c|c|c|c|c|}
\hline \multicolumn{2}{|c|}{ Caractères } & \multirow{2}{*}{$\begin{array}{l}\text { Effectifs } \\
\text { observés }\end{array}$} & \multirow{2}{*}{$\begin{array}{c}\chi^{2} \text { calculé } \\
\text { (disjonction } \\
\text { théorique } 3: 1 \text { ) }\end{array}$} & \multirow{2}{*}{$\begin{array}{c}\text { Probabilité } \\
\text { du } \chi^{2}\end{array}$} \\
\hline Dominant & Récessif & & & \\
\hline mâle-fertile & : mâle stérile & $17: 5$ & 0,061 & 0,81 \\
\hline chair blanche & : chair jaune & $12: 5$ & 0,176 & 0,67 \\
\hline fruit plat & : fruit rond & $9: 8$ & 4,412 & 0,04 \\
\hline fruit doux & : fruit acide & $11: 6$ & 0,961 & 0,33 \\
\hline
\end{tabular}

(1949) ; ils correspondent aux initiales de : Pollen stérile (Ps, ps), Yellow (Y, y), Saucer (S, s), Doux (D, d).

Le géniteur mâle que nous avons utilisé était la nectarine américaine «Independence ». Ce choix se justifiait par la belle présentation de ses fruits et par le fait qu'utilisant le caractère fruit à épiderme glabre, caractère mendélien récessif, il serait possible de le faire réapparaître en $\mathrm{F}_{2}$ et d'obtenir ainsi, par recombinaison, des nectarines plates. Nous avions autofécondé en 1973 la nectarine «Independence » et obtenu une toute petite famille de 10 arbres qui nous a permis toutefois d'observer que cette variété est hétérozygote pour la stérilité mâle ( 7 arbres étaient mâles fertiles et 3 mâles stériles) et homozygote récessive pour les caractères couleur de la chair (10 arbres donnant des fruits à chair jaune), forme du fruit (10 arbres à fruits ronds), saveur du fruit (10 arbres à fruits acides), comme on pouvait s'y attendre puisqu'ils sont récessifs. La formule génotypique d' "Independence» pour ces caractères est donc: Psps yy ss dd.

\section{III. ÉTUDE DE LA GÉNÉRATION HYBRIDE $F_{1}$}

Cette génération n'est pas tant intéressante par les progrès réalisés dans l'amélioration de l'aspect extérieur des pêches plates que par les études qu'elle permet de faire en génétique mendélienne. Malheureusement, l'effectif de la famille ( 24 arbres) est trop réduit pour que les conclusions qui seront faites puissent être considérées comme définitives.

La variété "Independence » étant récessive pour les caractères couleur de la chair, forme du fruit, saveur du fruit, son croisement avec la variété « Kiang Si » constitue un test cross. Si l'on considère séparément chacun de ces couples de caractères, les disjonctions observées confirment l'état hétérozygote de "Kiang Si » (tabl. 2).

Si l'on s'intéresse maintenant aux associations 2 par 2 de ces couples de caractères (tabl. 3), les effectifs observés pour les associations couleur de la chairforme du fruit, couleur de la chair-saveur du fruit sont ceux d'une disjonction indépendante. Au contraire des 2 précédentes, l'association forme du fruitsaveur du fruit a un $\chi^{2}$ dont la probabilité est faible $(0,31)$ dans le cas de l'hypothèse d'une disjonction 


\section{TABLEAU 2}

Disjonction des caractères couleur de la chair, forme du fruit, saveur du fruit sur les hybrides "Kiang Si » $\times$ " Independence 》. Segregation of the characters flesh colour, fruit shape and fruit taste in the hybrids « Kiang Si» x «Independence ».

\begin{tabular}{|c|c|c|c|c|}
\hline \multicolumn{2}{|c|}{ Caractères } & \multirow{2}{*}{$\begin{array}{l}\text { Effectifs } \\
\text { observés }\end{array}$} & \multirow{2}{*}{$\begin{array}{c}\chi^{2} \text { calculé } \\
\text { (disjonction } \\
\text { théorique } 1: 1 \text { ) }\end{array}$} & \multirow{2}{*}{$\begin{array}{l}\text { Probabilité } \\
\text { du } \chi^{2}\end{array}$} \\
\hline Dominant & Récessif & & & \\
\hline chair blanche & : chair jaune & $11: 13$ & 0,167 & 0,68 \\
\hline fruit plat & : fruit rond & $13: 11$ & 0,167 & 0,68 \\
\hline fruit doux* & : fruit acide & $12: 11$ & 0,043 & 0,83 \\
\hline
\end{tabular}

* L'acidité du fruit n'a pu être mesurée sur un arbre. indépendante $(1: 1: 1: 1)$. Cette faible probabilité ne peut pas s'expliquer par le manque d'accord des ségrégations fruit plat-fruit rond et fruit doux-fruit acide au rapport $1: 1$. Il peut s'expliquer par contre en supposant une liaison (linkage) entre les loci déterminant la forme du fruit et la saveur du fruit. Cette liaison pourrait être (en appliquant la règle du maximum de vraisemblance aux données que nous possédons) de 30 centimorgans. L'excès des associations fruit platfruit acide, fruit rond-fruit doux, indique que les gènes responsables de ces phénotypes sont en répulsion chez «Kiang Si ».

Nous avons jusqu'ici négligé volontairement le caractère stérilité mâle. Les 2 parents étant hétérozygotes pour ce caractère, leur croisement revient à créer

TABLEAU 3

Disjonction des associations couleur de la chair-forme du fruit, couleur de la chair-saveur du fruit, forme du fruit-saveur du fruit, dans la $F_{l}$ "Kiang $S i$ 》 « Independence ».

Segregation of the associations flesh colour-fruit shape, flesh colour-fruit taste, fruit shape-fruit taste in the $F_{1}$ " Kiang $S i 》 \times$ «Independence ".

\begin{tabular}{llccc}
\hline Phénotypes & $\begin{array}{c}\text { Effectifs } \\
\text { observés }\end{array}$ & $\begin{array}{c}\text { Effectifs } \\
\text { théoriques }\end{array}$ & $\begin{array}{c}\chi^{2} \text { calculé } \\
\text { (disjonction } \\
\text { théorique } 1: 1: 1: 1 \text { ) }\end{array}$ & $\begin{array}{c}\text { Probabilité } \\
\text { du } \chi^{2}\end{array}$ \\
\hline $\begin{array}{llll}\text { chair blanche } \\
\text { chair blanche }\end{array}$ & $:$ fruit plat & 7 & 6 & 0,999 \\
chair jaune & $:$ fruit rond & 4 & 6 & \\
chair jaune & $:$ fruit rond & 6 & 6 & \\
chair blanche & $:$ fruit doux & 7 & 6 & \\
chair blanche & $:$ fruit acide & 6 & 5,75 & \\
chair jaune & $:$ fruit doux & 5 & 5,75 & \\
chair jaune & $:$ fruit acide & 6 & 5,75 & \\
fruit plat & $:$ fruit doux & 6 & 5,75 & \\
fruit plat & $:$ fruit acide & 4 & 5,75 & \\
fruit rond & $:$ fruit doux & 8 & 5,75 & \\
fruit rond & $:$ fruit acide & 8 & 5,75 & \\
\hline
\end{tabular}

TABLEAU 4

Disjonction des différentes combinaisons de caractères stérilité mâle-couleur de la chair, stérilité mâle-forme du fruit, stérilité mâle-saveur du fruit, sur les hybrides $F_{1}$ "Kiang $S i » \times$ «Independence ».

Segregation of different combinations of the characters pollen sterility-flesh colour, pollen sterility-fruit shape, pollen sterility-fruit taste, in the hybrids $F_{I}$ «Kiang Si » $\times$ «Independence ».

\begin{tabular}{|c|c|c|c|c|c|}
\hline & Phénotypes & $\begin{array}{l}\text { Effectifs } \\
\text { observés }\end{array}$ & $\begin{array}{c}\text { Effectifs } \\
\text { théoriques } \\
\text { (disjonction } 3: 3: 1: 1 \text { ) }\end{array}$ & $\chi^{2}$ & $\begin{array}{l}\text { Probabilité } \\
\text { du } \chi^{2}\end{array}$ \\
\hline mâle fertile & : chair blanche & 8 & 9 & 0,222 & 0,97 \\
\hline mâle fertile & : chair jaune & 10 & 9 & & \\
\hline mâle stérile & : chair blanche & 3 & 3 & & \\
\hline mâle stérile & : chair jaune & 3 & 3 & & \\
\hline mâle fertile & : pêche plate & 10 & 9 & 0,222 & 0,97 \\
\hline mâle fertile & : pêche ronde & 8 & 9 & & \\
\hline mâle stérile & : pêche plate & 3 & 3 & & \\
\hline mâle stérile & : pêche ronde & 3 & 3 & & \\
\hline mâle fertile & : fruit doux* & 8 & 8,63 & 0,768 & 0,86 \\
\hline mâle fertile & : fruit acide & 9 & 8,63 & & \\
\hline mâle stérile & : fruit doux & 4 & 2,87 & & \\
\hline mâle stérile & : fruit acide & 2 & 2,87 & & \\
\hline
\end{tabular}

* L'acidité des fruits d'un arbre n'a pu être mesurée. 
une $F_{2}$. De fait, nous avons trouvé dans cette famille 18 arbres mâle fertiles et 6 arbres mâle stériles.

Intéressons-nous par ailleurs aux associations suivantes :

Stérilité mâle-couleur de la chair.

Stérilité mâle-forme du fruit.

Stérilité mâle-saveur du fruit.

Si ces caractères se disjoignent indépendamment, nous devrions trouver les proportions phénotypiques 3:3:1:1 ; le tableau 4 confronte les effectifs observés aux effectifs théoriques pour ces différentes associations. Le faible écart entre les unes et les autres plaide en faveur d'une absence de liaison entre ces caractères (ou une liaison à plus de 50 centimorgans). Nous avons déjà établi ce résultat pour la première de ces associations (MONET \& BASTARD, 1983).

Certes, cette famille $\mathrm{F}_{1}$ paraît quelquefois trop conforme aux prévisions; nous sommes persuadés pourtant qu'il ne s'agit pas d'un échantillon biaisé et que l'on fera les mêmes conclusions si l'on refait de tels croisements.

\section{IV. ÉTUDE SUCCINCTE DE LA GÉNÉRATION $F_{2}$}

Nous avons autofécondé 8 arbres à fruits plats sur 12 de la génération $F_{1}$ et délaissé volontairement les arbres à fruits ronds. Notre objectif étant l'amélioration des pêches plates, seuls les individus portant ce caractère étaient susceptibles d'en redonner puisque le caractère est dominant .

Les familles issues de ces 8 arbres ont été cultivées séparément.

Par suite de la taille réduite des familles et de l'absence de production de certains arbres, nous ne sommes pas en mesure de confirmer les observations faites dans la $F_{1}$ et notamment la présence d'une liaison entre les caractères forme du fruit et saveur du fruit. Pour celle-ci, 4 familles (4-8-10-12) étaient susceptibles de nous donner une nouvelle réponse, mais les faibles effectifs observés ne permettent aucune conclusion même en regroupant les classes des 4 familles (tabl. 5).

TABLEAU 5

Disjonction des différentes combinaisons de caractères forme du fruit-saveur du fruit sur 4 familles de la génération $F_{2}$ du croisement “Kiang Si » $\times$ « Independence ».

Segregation of different combinations of characters fruit shape-fruit taste in $4 \mathrm{~F}_{2}$ families of the cross « Kiang $\mathrm{Si}$ 》 $\times$ «Independence ».

\begin{tabular}{|c|c|c|c|c|}
\hline \multirow{3}{*}{$\begin{array}{c}\text { Numéro } \\
\text { des familles }\end{array}$} & \multicolumn{4}{|c|}{ Phénotypes observés } \\
\hline & \multicolumn{2}{|c|}{ Plat } & \multicolumn{2}{|c|}{ Rond } \\
\hline & Doux & acide & Doux & acide \\
\hline 4 & 4 & 1 & 3 & 3 \\
\hline 8 & - & 1 & 3 & - \\
\hline 10 & 1 & 1 & 2 & 2 \\
\hline 12 & 5 & 1 & - & - \\
\hline Total & 10 & 4 & 8 & 5 \\
\hline Effectifs théoriques* & $16,8 i$ & 3,44 & 3,44 & 3,31 \\
\hline
\end{tabular}

Si du point de vue génétique cette génération $F_{2}$ (non entièrement observée à ce jour) n'apporte pas les informations que l'on aurait pu souhaiter, elle est beaucoup plus intéressante quant aux progrès réalisés sur l'amélioration des pêches plates. D'abord, comme nous le souhaitions, nous avons obtenu des nectarines plates (fig. 1). Ce nouveau type de fruit, non connu à l'état naturel, rassemble les caractères fruit plat et fruit glabre; nous proposons de l'appeler «Platerine ». Parmi les descendants donnant des pêches plates, certains avaient des fruits ayant une présentation sensiblement améliorée (fig. 2). Deux d'entre eux (les arbres 5 de la famille 4 et 5 de la famille 12) ont été présélectionnés et feront l'objet d'une expérimentation supplémentaire en vue d'une demande d'inscription au Catalogue officiel.

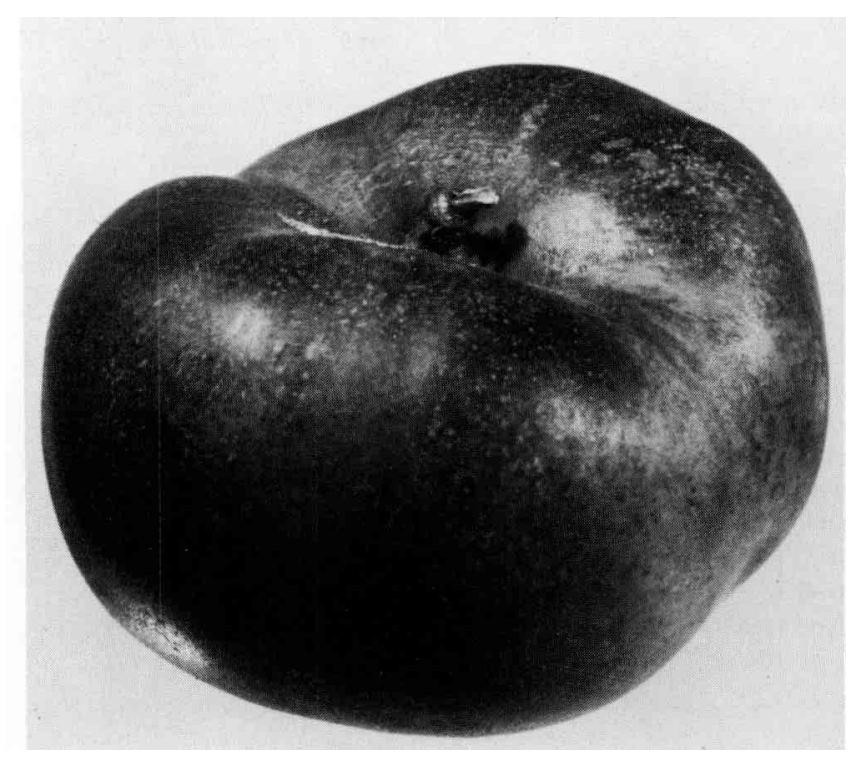

Figure 1

Nectarine plate (Platerine) à chair blanche apparue dans la génération $F_{2}$.

Flat nectarine (Platerine) with white flesh obtained in the $F_{2}$.

\section{CONCLUSIONS}

Nous sommes en présence d'un cas d'amélioration relativement simple dans lequel il s'agit de maintenir 2 caractères (fruit plat et doux) à hérédité simple (monogénique dominant) alors que les autres caractères à améliorer (couleur de l'épiderme du fruit, sensibilité aux craquelures) sont à hérédité quantitative. Dès la $2^{\mathrm{e}}$ génération, les progrès sont importants. Par rétrocroisements successifs, nous devrions progresser encore. Nous avons commencé ceux-ci sur les meilleurs descendants de la génération $F_{2}$ en utilisant un nouveau géniteur nectarine. 
Grâce aux précautions que nous avons prises lors de la réalisation des hybridations ou des autofécondations, grâce aussi au suivi généalogique de chaque individu, nous avons pu faire de nouvelles études de génétique mendélienne chez le pêcher. Bien que néces- sitant une confirmation, elles devraient contribuer à accroître la connaissance génétique de cette espèce.

Reçu le 15 novembre 1984. Accepté le 4 avril 1985.

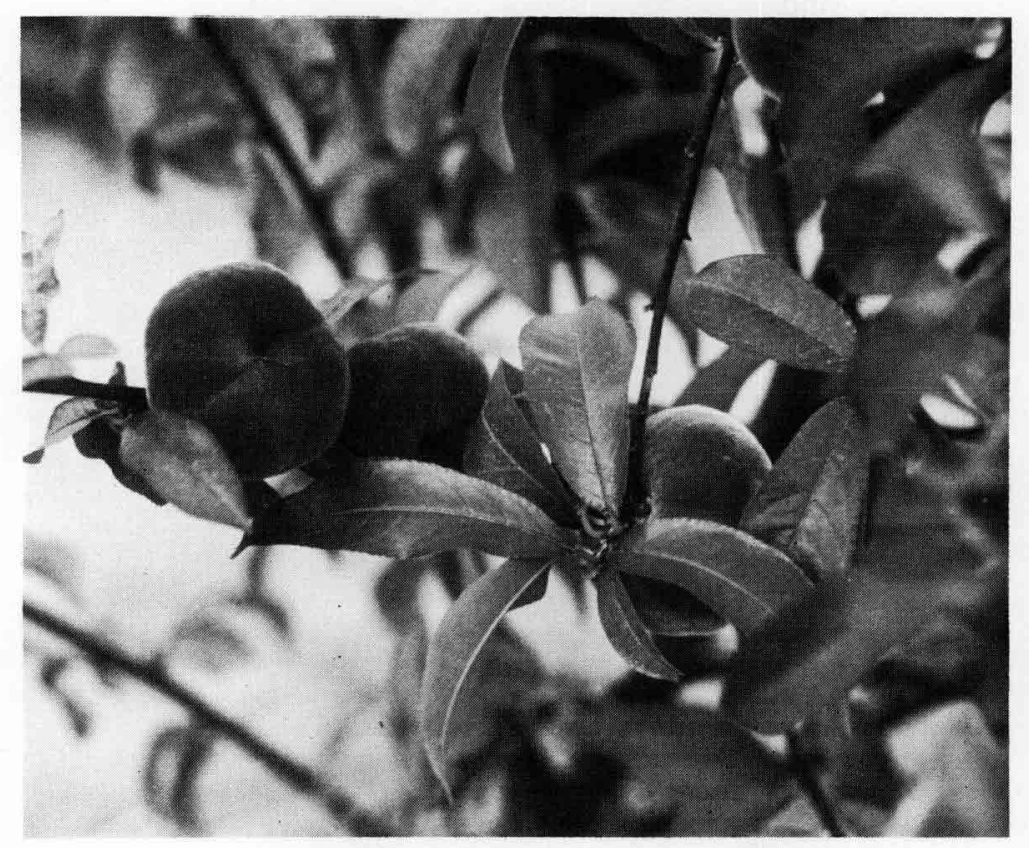

Figure 2

Fruits obtenus en serre sur des plants greffés issus de l'arbre 5 de la famille 4. On remarque la couleur rouge homogène de l'épiderme et l'absence de craquelures.
Fruits obtained in the greenhouse on grafted trees originating from tree number 5 of family 4. Note homogeneous red skin colour and the absence of cracking.

\section{RÉFÉRENCES BIBLIOGRAPHIQUES}

Bailey J. S., French A. P., 1949. The inheritance of certain fruit and foliage characters in the peach. Mass. Agr. Exp. Sta. Bull., 452, 1-31.

Carriere E. A., 1868. Description et classification des variétés de pêchers et brugnonniers. Zanote, Montereau, $103 \mathrm{p}$.

Hedrick U. P., 1917. The peaches of New York. Lyon Company Albany, $541 \mathrm{p}$.

Lesley J. W., 1939. A genetic study of saucer fruit shape and other characters in the peach. Proc. Am. Soc. Hortic. Sci., 37, 218-222.
Monet R., 1979. Transmission génétique du caractère " fruit doux » chez le pêcher, incidence sur la sélection pour la qualité. $C . R$. Symp. Section Fruits EUCARPIA : Amélioration des arbres fruitiers, Angers, I.N.R.A. éd., 273-277.

Monet R., Bastard Y., 1983. Nouveaux cas de ségrégation indépendante de caractères mendéliens chez le pêcher. Agronomie, 3 (4), 387-390.

Weldon G. P., Lesley J. W., 1933. The babcock peach. Coll. Agric. Agric. Exp. Stat., Berkeley, California, circ, 328. 